\title{
Perfil epidemiológico de sífilis gestacional no Nordeste Brasileiro
}

\author{
Epidemiological profile of gestational syphilis in Northeast Brazill \\ Perfil epidemiológico de la sífilis gestacional en el Noreste de Brasil
}

Recebido: 20/08/2021 | Revisado: 27/08/2021 | Aceito: 31/08/2021 | Publicado: 03/09/2021

\author{
Ana Karolina dos Santos Ferreira \\ ORCID: https://orcid.org/0000-0002-0576-702X \\ Centro Universitário Mario Pontes Jucá, Brasil \\ E-mail: karolinaferreirafs@gmail.com \\ Mariana Quintiliano Wanderley Sandes \\ ORCID: https://orcid.org/0000-0002-3716-6528 \\ Centro Universitário Mario Pontes Jucá, Brasil \\ E-mail: mariliswanderley@hotmail.com \\ Janinne Santos de Melo \\ ORCID: https://orcid.org/0000-0001-8049-6891 \\ Hospital Memorial Arthur Ramos, Brasil \\ E-mail: janinnesantosmelo@ hotmail.com \\ Paulo César de Almeida \\ ORCID: https://orcid.org/0000-0002-2867-802X \\ Universidade Estadual do Ceará, Brasil \\ E-mail: pc2015almeida@gmail.com \\ Clodis Maria Tavares \\ ORCID: https://orcid.org/0000-0001-6804-3064 \\ Universidade Federal de Alagoas, Brasil \\ E-mail: clodistavares@yahoo.com.br \\ Tamyssa Simões dos Santos \\ ORCID: https://orcid.org/0000-0002-7911-0389 \\ Centro Universitário Mario Pontes Jucá, Brasil \\ E-mail: simoestamyssa@gmail.com
}

\begin{abstract}
Resumo
Objetivou-se analisar dados sociodemográficos e clínicos de sífilis gestacional no nordeste brasileiro. Estudo ecológico, de abordagem quantitativa, realizado a partir de dados secundários do Sistema de Informação de Agravos de Notificação (SINAN), disponibilizados pelo Departamento de Informática do Sistema Único de Saúde (DATASUS) referente aos casos de sífilis gestacional no nordeste brasileiro durante os anos de 2015 a 2019. Verificou-se um aumento no número de casos de sífilis em gestantes entre as faixas etárias de 20 a 29 anos em todos os anos analisados $(53,5 \%)$. A maioria das gestantes possuíam $5^{\mathrm{a}}$ a $8^{\mathrm{a}}$ série incompleta $(23,3 \%)$, com predominância na raça parda $(68,6 \%)$. Houve significativo aumento do número de casos ao longo do período nos estados brasileiros, sendo o menor crescimento na Paraíba $(0,73 \%)$ e o maior na Bahia $(1,91 \%)$. Pôde-se notar que a incidência de sífilis gestacional em mulheres jovens vem aumentando de forma gradual no Nordeste, para tanto, se faz necessário intervenções mais eficazes para o controle da doença, a fim de que ocorra cada vez mais uma redução dos casos de transmissão vertical e complicações fetais.
\end{abstract}

Palavras-chave: Epidemiologia; Sífilis; Gravidez; Gestantes; Cuidado pré-natal.

\begin{abstract}
The objective was to analyze sociodemographic and clinical data on gestational syphilis in northeastern Brazil. An ecological study, with a quantitative approach, based on secondary data from the Information System for Notifiable Diseases (SINAN), made available by the Information Technology Department of the Unified Health System (DATASUS) regarding cases of gestational syphilis in northeastern Brazil during the years from 2015 to 2019 . There was an increase in the number of syphilis cases in pregnant women aged 20 to 29 years in all years analyzed (53.5\%). Most pregnant women had incomplete 5th to 8th grade $(23.3 \%)$, with a predominance of the brown race $(68.6 \%)$. There was a significant increase in the number of cases over the period in the brazilians states, with the lowest growth in Paraíba $(0.73 \%)$ and the highest in Bahia $(1.91 \%)$. It could be noted that the incidence of gestational syphilis in young women has been increasing gradually in the Northeast, therefore, more effective interventions are needed to control the disease so that there is an increasingly reduction in cases of vertical and fetal complications.
\end{abstract}

Keywords: Epidemiology; Syphilis; Pregnancy; Pregnant women; Prenatal care. 


\section{Resumen}

El objetivo fue analizar datos sociodemográficos y clínicos sobre la sífilis gestacional en el noreste de Brasil. Estudio ecológico, con enfoque cuantitativo, basado en datos secundarios del Sistema de Información de Enfermedades de Notificación (SINAN), puesto a disposición por el Departamento de Tecnología de la Información del Sistema Único de Salud (DATASUS) sobre casos de sífilis gestacional en el noreste de Brasil durante los años de 2015 a 2019 . Hubo un aumento en el número de casos de sífilis en gestantes entre los grupos de edad de 20 a 29 años en todos los años analizados $(53,5 \%)$. La mayoría de las embarazadas presentaban de $5^{\circ}$ a $8^{\circ}$ grado incompleto $(23,3 \%)$, con predominio de la raza morena $(68,6 \%)$. Hubo un aumento significativo en el número de casos durante el período en los estados brasileños, con el menor crecimiento en Paraíba (0,73\%) y el mayor en Bahía $(1,91 \%)$. Cabe señalar que la incidencia de sífilis gestacional en mujeres jóvenes ha ido aumentando gradualmente en el Nordeste, por lo tanto, se necesitan intervenciones más efectivas para controlar la enfermedad, de manera que haya una reducción cada vez mayor de los casos de transmisión vertical y complicaciones fetales.

Palabras clave: Epidemiología; Sífilis; El embarazo; Mujeres embarazadas; Cuidado prenatal.

\section{Introdução}

A sífilis, também conhecida como cancro duro, é uma infecção bacteriana sistêmica, de evolução crônica, causada pelo Treponema pallidum, bactéria gram-negativa, do grupo das espiroquetas, de alta patogenicidade. Quando não tratada progride ao longo de muitos anos, sendo classificada em sífilis primária, secundária, latente recente, latente tardia e terciária. Seu período de incubação varia de 10 a 90 dias (média de 21 dias) a partir do contato sexual infectante (Brasil, 2019).

A via sexual é a principal forma de transmissão da infecção, que dá origem à forma adquirida. A sífilis gestacional se apresenta em mulheres grávidas com a enfermidade, que não foram tratadas ou que passaram por tratamento inadequado, a infecção pode ocasionar a forma congênita, a qual a bactéria é transmitida por via transplacentária ao concepto (Terra, 2019).

As manifestações da doença estão interligadas com os estágios e o tempo da infecção. Por este motivo, os sinais e sintomas alternam períodos de atividade com características clínicas, imunológicas e histopatológicas distintas (sífilis primária, secundária e terciária) com períodos de latência (Souza; Rodrigues; Gomes, 2019).

Estima-se que no mundo ocorram mais de 11 milhões de novos casos de sífilis por ano, com altas taxas de incidência em países da América Latina, África e Ásia. No Brasil, a doença persiste como um grave problema de saúde pública, principalmente na população materno-infantil (Lima et al., 2019).

No período de 2005 a junho de 2020, foram notificados no Sistema de Informação de Agravos de Notificação (SINAN) 384.411 casos de sífilis em gestantes, dos quais 45,3\% eram residentes na região Sudeste, 20,9\% na região Nordeste, 14,8\% na região Sul, 10,2\% na região Norte e 8,8\% na região Centro-Oeste (Brasil, 2020).

Quando analisado o período gestacional de detecção de sífilis, segundo Unidade de Federação (UF) e capitais brasileiras, observou-se que, em 2019 a maior proporção das mulheres $(38,7 \%)$ foi diagnosticada no primeiro trimestre, ao passo que 24,2\% representaram diagnósticos realizados no segundo trimestre, e 30,4\% no terceiro trimestre de gestação (Brasil, 2017).

Embora todas as faixas etárias consideradas como idade reprodutiva feminina contenham números expressivos para novos casos e diagnósticos de sífilis em diferentes fases da doença, o boletim epidemiológico do Ministério da Saúde, considerando o ano de 2019, aponta que 55,7\% das gestantes diagnosticadas com sífilis encontravam-se na faixa etária de 20 a 29 anos (Brasil, 2017).

Sabe-se que a sífilis é uma infecção de tratamento acessível, sendo importante que a gestante contaminada passe por diagnóstico no pré-natal e seja submetida ao tratamento adequado juntamente com seu parceiro; caso isso não ocorra, acarretará em abortos, partos prematuros e sequelas para o bebê. Quanto mais cedo for iniciada a terapia medicamentosa, melhores resultados serão alcançados (Oliveira et al., 2019).

Quando não há tratamento adequado, a sífilis gestacional pode ocasionar consequências negativas ao feto, resultando em sífilis congênita; com isso, as sequelas para o bebê vão desde efeitos adversos na gestação como aborto espontâneo, baixo 
peso ao nascer, até o aparecimento dos seguintes achados pós-parto: erupções cutâneas, anemia, icterícia, leucopenia ou leucocitose, hepatoesplenomegalia, síndrome nefrótica, entre outros (Oliveira et al., 2019).

Neste contexto, a região Nordeste ocupa o segundo lugar do ranking das regiões com maior número de casos de sífilis em gestantes no País, sendo o estado do Maranhão o que apresenta a maior taxa de incidência dessa região, com seis casos por mil nascidos vivos, concentrando as maiores taxas em São Luís, capital do estado (Conceição; Câmara; Pereira, 2019).

Assim, o objetivo deste estudo consiste em analisar dados sociodemográficos e clínicos de sífilis gestacional no nordeste brasileiro, além de realizar o levantamento de dados sociodemográficos e descrevê-los. Desta forma, surge a seguinte pergunta de pesquisa: qual o perfil sociodemográfico da sífilis gestacional no nordeste brasileiro entre 2015 e 2019 ?

Como hipótese, acredita-se que a sífilis gestacional ainda é prevalente no nordeste brasileiro, devido ao fato de um expressivo número de mulheres não receberem acompanhamento nas Unidades Básicas de Saúde (UBS) durante consultas de pré-natal.

\section{Metodologia}

Estudo ecológico, de abordagem quantitativa, realizado a partir de dados secundários do SINAN, disponibilizados pelo Departamento de Informática do Sistema Único de Saúde (DATASUS) referente aos casos de sífilis gestacional no nordeste brasileiro em um período compreendido entre 2015 a 2019.

Foram analisadas as variáveis faixa etária, escolaridade, raça/cor da pele, esquema de tratamento, classificação clínica, realização de pré-natal, momento do diagnóstico e adequação de tratamento ao longo dos anos estudados.

Como critérios de inclusão deste estudo têm-se gestantes portadoras de sífilis, residentes na região nordeste do país, de todas as idades e níveis econômicos; já como critérios de exclusão têm-se gestantes residentes no nordeste, mas com moradia fixa em outro país.

Apesar de ser uma pesquisa envolvendo dados sobre seres humanos, não foi necessária a avaliação de um comitê de ética, tendo em vista que as informações estão depositadas no Sistema de Informação de Agravos de Notificação (SINAN) para pesquisa pública, onde não há identificação pessoal de nenhum dos participantes.

A comparação da tendência de proporções do número de casos de sífilis segundo as variáveis foi realizada pelo teste de Qui-quadrado para tendência em proporção. Compararam-se as médias das taxas segundo a faixa etária pelo teste ANOVA, sendo as comparações múltiplas analisadas pelo teste de Games-Howell. Ainda fez-se a regressão linear ao longo do período de 2015 a 2019, do número de casos de sífilis total e por período de diagnóstico da doença (pré-natal, parto e pós-parto).

Foram consideradas como estatisticamente significantes as análises com $\mathrm{p}<0,05$. Os dados foram processados no SPSS 20.0, licença número 10101131007.

\section{Resultados}

Os dados referentes à sífilis gestacional de acordo com a faixa etária apresentaram um aumento entre as faixas etárias de 20 a 29 anos em todos os anos de 2015-2019. Em 2015 com 51,4\% dos casos e em 2019 esse número aumentou para 53,5\% (Tabela 1).

Com relação à escolaridade, pôde-se perceber que o maior índice de casos de sífilis gestacional apresentou-se em mulheres com $5^{\mathrm{a}}$ a $8^{\mathrm{a}}$ série incompleta, com cerca de 23,3\% dos casos no ano de 2018.

Levando em consideração o tipo de raça, a que se apresentou com maior percentual de sífilis gestacional na região nordeste foi a parda $(35,2 \%)$ no ano de 2018 . Em contrapartida a raça que menos predominou foi a indígena com 5,2\% dos casos em 2017. 
Analisando-se o número de casos, houve uma tendência decrescente ao longo do período de 2015 a 2019 (p=0,007). Essa mesma tendência de sífilis dentro de cada faixa etária encontrou-se dentro de cada nível de escolaridade (p<0,0001) e dentro de cada categoria de raça/cor $(\mathrm{p}=0,031)$.

Tabela 1 - Distribuição de casos de sífilis em gestantes segundo aspectos sociodemográficos. Nordeste, 2015 a 2019.

\begin{tabular}{|c|c|c|c|c|c|}
\hline Variável & $\begin{array}{c}2015 \\
\mathrm{~N}(\%)\end{array}$ & $\begin{array}{l}2016 \\
\mathrm{~N}(\%) \\
\end{array}$ & $\begin{array}{c}2017 \\
\mathrm{~N}(\%)\end{array}$ & $\begin{array}{l}2018 \\
\mathrm{~N}(\%) \\
\end{array}$ & $\begin{array}{l}2019 \\
\mathrm{~N}(\%) \\
\end{array}$ \\
\hline $\begin{array}{c}\text { Faixa etária (ano) } \\
10 \text { a } 14\end{array}$ & $100(1,7)$ & $98(1,5)$ & $159(1,7)$ & $183(1,2)$ & $\begin{array}{l}\text { p 0,007 } \\
66(1,2)\end{array}$ \\
\hline 15 a 19 & $1.472(24,7)$ & $1.734(26,3)$ & $2.339(25,4)$ & $3.628(24,7)$ & $1.357(24,0)$ \\
\hline 20 a 29 & $3.065(51,4)$ & $3.347(50,8)$ & $4.789(52,0)$ & $7.606(51,7)$ & $3.024(53,5)$ \\
\hline 30 a 39 & $1.185(19,9)$ & $1.269(19,3)$ & $1.742(18,9)$ & $2.959(20,1)$ & $1.093(19,3)$ \\
\hline 40 ou mais & $138(2,3)$ & $140(2,1)$ & $180(2)$ & $327(2,2)$ & $116(2,1)$ \\
\hline $\begin{array}{l}\text { Escolaridade } \\
\text { Analfabeto }\end{array}$ & $95(1,6)$ & $90(1,4)$ & $103(1,1)$ & $131(0,9)$ & $\begin{array}{l}<0,0001 \\
60(1,1)\end{array}$ \\
\hline $\begin{array}{l}1^{\mathrm{a}} \text { a } 4^{\mathrm{a}} \text { série } \\
\text { incompleta }\end{array}$ & $536(9,0)$ & $537(8,1)$ & $666(7,2)$ & $940(6,4)$ & $325(5,7)$ \\
\hline $4^{a}$ série completa & $303(5,1)$ & $364(5,5)$ & $427(4,6)$ & $591(4,0)$ & $255(4,5)$ \\
\hline $\begin{array}{l}5^{\mathrm{a}} \text { a } 8^{\mathrm{a}} \text { série } \\
\text { incompleta }\end{array}$ & $1.352(22,7)$ & $1.427(21,6)$ & $2.058(22,3)$ & $3.425(23,3)$ & $1.307(23,1)$ \\
\hline $\begin{array}{l}\text { Fundamental } \\
\text { completo }\end{array}$ & $476(8,0)$ & $553(8,4)$ & $778(8,4)$ & $1.230(8,4)$ & $486(8,6)$ \\
\hline Médio Incompleto & $649(10,9)$ & $734(11,1)$ & $1.147(12,5)$ & $1.822(12,4)$ & $719(12,7)$ \\
\hline Médio Completo & $712(11,9)$ & $832(12,6)$ & $1.434(15,6)$ & $2.586(17,6)$ & $1.038(18,3)$ \\
\hline Superior incompleto & $51(0,9)$ & $45(0,7)$ & $90(1,0)$ & $131(0,9)$ & $55(1,0)$ \\
\hline Superior Completo & $28(0,5)$ & $42(0,6)$ & $67(0,7)$ & $135(0,9)$ & $44(0,8)$ \\
\hline $\begin{array}{ll}\text { Raça } & \\
& \text { Branca }\end{array}$ & $619(10,4)$ & $686(10,4)$ & $1.000(10,9)$ & $1.600(10,9)$ & $\begin{array}{c}0,031 \\
720(12,7)\end{array}$ \\
\hline Preta & $777(13,0)$ & $1.001(15,2)$ & $1.241(13,5)$ & $1.758(12,0)$ & $514(9,1)$ \\
\hline Amarela & $64(1,1)$ & $54(0,8)$ & $105(1,1)$ & $154(1,0)$ & $69(1,2)$ \\
\hline Parda & $4.029(67,6)$ & $4.314(65,4)$ & $6.238(67,7)$ & $10.085(68,6)$ & $3.967(70,1)$ \\
\hline Indígena & $27(0,5)$ & $36(0,5)$ & $38(0,4)$ & $54(0,4)$ & $27(0,5)$ \\
\hline
\end{tabular}

Fonte: SINAN/Ministério da Saúde/Brasil, 2020. Teste de Qui-quadrado para tendência em proporção.

Quando comparadas as médias de faixas etárias, a menor taxa referente aos casos de sífilis em gestantes foi encontrada na faixa etária de 10 a 14 anos $(1,74 \pm 0,78)$. A maior taxa foi a da faixa de 20 a 29 anos $(51,37 \pm 3,18)$, sendo diferente das demais taxas (Tabela 2). 
Tabela 2 - Comparação da média das taxas de sífilis, segundo a faixa etária, Nordeste, 2015 a 2019.

$\begin{array}{lll}\text { Faixa etária } & \text { Média } \pm \text { DP } & \text { Comparações múltiplas } \\ & & \\ 10-14^{1} & 1,74 \pm 0,78 & \text { Faixa } 1 \text { diferiu } 2,3,4 \text { e } 5 \\ 15-19^{2} & 51,37 \pm 3,18 \quad \text { Faixa 3diferiu da } 4 \text { e } 5 \\ 20-29^{3} & 19,11 \pm 3,50 \quad \text { Faixa } 4 \text { diferiu da } 5 \\ 30-39^{4} & 6,34 \pm 18,50 & \\ 40 \text { ou }+^{5} & \end{array}$

Fonte: $\mathrm{p}$ do teste ANOVA <0,0001. Comparações múltiplas pelo teste de Games-Howell. Taxa por 1000 nascidos vivos.

Ao analisar as taxas de casos de sífilis em gestantes pelos estados do Nordeste brasileiro, pôde-se perceber que houve um significativo aumento do número de casos ao longo do período nos estados $(\mathrm{p}<0,05)$, sendo o menor crescimento na Paraíba $(b=0,73 ; p=0,020)$ e o maior na Bahia $(b=1,91 ; p<0,0001)$ (Tabela 3). Com relação ao período de diagnóstico da sífilis no pré-natal, as taxas também cresceram nos estados, excetuando o Piauí $(b=0,53 ; p=0,228)$ e Maranhão $(b=0,81 ; p=0,061)$. Houve decréscimo no número de casos quanto ao diagnóstico no momento do parto em Alagoas ( $b=-1,91 ; p=0,001)$, Maranhão $(\mathrm{b}=-1,30 ; \mathrm{p}=0,026)$, Pernambuco $(\mathrm{b}=-1,49 ; \mathrm{p}=0,007)$ e Piauí $(\mathrm{b}=-1,10 ; \mathrm{p}=0,031)$. Já no pós- parto o número de casos diminuiu somente na Bahia $(b=-1,98 ; \mathrm{p}<0,0001)$ e Paraíba $(b=-1,28 ; b=0,030)$ (Tabela 3).

Tabela 3 - Análise de regressão linear da taxa de casos de sífilis, segundo o período de diagnóstico, Nordeste, 2015 a 2019.

\begin{tabular}{|c|c|c|c|c|c|c|c|c|c|}
\hline \multirow{2}{*}{$\begin{array}{l}\text { Total } \\
\text { Estado }\end{array}$} & \multirow{2}{*}{\multicolumn{2}{|c|}{$\begin{array}{c}\text { pré-natal } \\
\qquad b^{\mathrm{a}}\end{array}$}} & \multicolumn{2}{|c|}{ parto } & \multicolumn{2}{|c|}{ pós-parto } & \multirow[b]{2}{*}{$\mathrm{p}$} & \multirow[b]{2}{*}{$\mathrm{b}$} & \multirow[b]{2}{*}{$\mathrm{p}$} \\
\hline & & & $\mathrm{p}$ & $\mathrm{b}$ & $\mathrm{p}$ & $\mathrm{b}$ & & & \\
\hline Alagoas & 1,61 & 0,003 & 2,01 & $<0,0001$ & $-1,91$ & 0,001 & $-0,27$ & 0,511 & \\
\hline Bahia & 1,91 & $<0,0001$ & 2,72 & $<0,0001$ & 0,47 & 0,262 & $-1,98$ & $<0,0001$ & \\
\hline Ceará & 1,27 & 0,004 & 1,05 & $\mathbf{0 , 0 3 8}$ & $-0,31$ & 0,285 & $-0,66$ & $\mathbf{0 , 0 8 7}$ & \\
\hline Maranhão & 1,54 & 0,001 & 0,81 & 0,061 & $-1,30$ & 0,026 & 0,16 & $\mathbf{0 , 7 7 3}$ & \\
\hline Paraíba & 0,73 & 0,020 & 1,63 & $<0,0001$ & 0,06 & 0,901 & $-1,28$ & $\mathbf{0 , 0 3 0}$ & \\
\hline Pernambuco & 1,84 & $\mathbf{0 , 0 0 7}$ & 1,09 & 0,009 & $-1,49$ & $\mathbf{0 , 0 0 7}$ & 0,55 & 0,446 & \\
\hline Piauí & 1,38 & 0,001 & 0,53 & 0,228 & $-1,10$ & $\mathbf{0 , 0 3 1}$ & 0,41 & 0,460 & \\
\hline Rio G Norte & 1,24 & 0,019 & 2,18 & 0,004 & $-0,00$ & 0,999 & $-2,04$ & 0,163 & \\
\hline Sergipe & 1,16 & 0,007 & 2,10 & $\mathbf{0 , 0 2 7}$ & $-1,58$ & 0,185 & 0,31 & 0,845 & \\
\hline
\end{tabular}

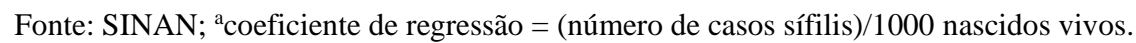

$\mathrm{Na}$ Tabela 4, pôde-se perceber que houve decréscimo em relação à sífilis primária e terciária, entretanto, ocorreu crescimento na classificação de sífilis secundária e latente $(\mathrm{p}<0,0001)$. No quesito realização de pré-natal, têm-se expressivo crescimento de gestantes que o realizaram em comparação com as que não realizaram $(\mathrm{p}<0,0001)$.

De acordo com o esquema de tratamento da sífilis gestacional, grande maioria das mulheres com a patologia no ano de 2019 , cerca de $87,2 \%$ dos casos, fizeram o uso da penicilina como tratamento de escolha e apenas $2,5 \%$ das mulheres 
realizaram outro tipo de tratamento; ao longo dos anos o número de uso da penicilina demonstrou aumento, quando comparados a outro tipo de tratamento. Em relação à adequação do tratamento, certificou-se que ele foi significativamente adequado durante os anos analisados $(\mathrm{p}<0,0001)$ (Tabela 4).

Foram comparados os dados referentes ao momento do diagnóstico de sífilis, e como resultados obteve-se um número expressivo na taxa média de casos diagnosticados no pré-natal, maior crescimento das taxas com passar dos anos no pós parto; durante o pré-natal e no momento do parto/curetagem obtiveram decréscimo $(\mathrm{p}<0,0001)$ (Tabela 4).

Tabela 4 - Distribuição de Casos de Sífilis em gestantes segundo aspectos clínicos e tratamento. Nordeste, 2015 a 2019.

\begin{tabular}{lrrrr}
\hline Esquema de Tratamento & & & & \\
Penicilina & $5.40(84,6)$ & $5.723(86,8)$ & $8.036(87,2)$ & $12.740(86,6)$ \\
Outro & $214(3,6)$ & $162(2,5)$ & $371(4,0)$ & $366(2,5)$
\end{tabular}

\section{Classificação Clínica}

Sífilis primária $2.058(34,5)$

$2.174(33,0)$

$2.932(31,8)$

$4.241(28,8)$

$1.613(28,5)$

Sífilis secundária

$351(5,9)$

$448(6,8)$

$546(5,9)$

$952(6,5)$

$400(7,1)$

Sífilis terciária

$728(12,2)$

$958(14,5) \quad 1.266(13,7)$

$1.733(11,8)$

$645(11,4)$

Sífilis latente

$$
1.088(18,3)
$$

$1.083(16,4) \quad 1.871(20,3)$

$3.733(25,4)$

$1.455(25,7)$

\section{Realização Pré Natal}

Sim

4.652(77,1)

$4.782(80,3)$

$5,640(80,9)$

$6.508(82,4)$

$2.680(83,6)$

Não

$865(14,3)$

$770(12,9)$

$864(12,4)$

927(11,7)

$289(9,0)$

\section{Momento de Diagnóstico}

Durante pré-natal

$2.538(42,1)$

$2.858(48,0)$

$3.405(48,8)$

$4.250(53,8)$

$1.808(56,4)$

Parto/curetagem

$2.453(40,7)$

$2.115(35,5)$

$2.519(36,1)$

$2.647(33,5)$

$1.084(33,8)$

Pós-parto

$620(10,3)$

691(11,6)

$694(10,0)$

$570(7,2)$

$174(5,4)$

\section{Adequaçãoao Esquema de Tratamento}

$<0,0001^{(1)}$ 
situações: a primeira é que Paraíba obteve a menor taxa de detecção no ano de 2016 e a segunda é que logo após o pico em 2018 houve um declínio dessas taxas em todos os estados em 2019.

Observou-se também que 2018 foi o ano com maior número em todos os Estados do Nordeste, sendo o período entre 2015 a 2017 sem oscilações significativas e o ano de 2019 sendo o que registrou a menor taxa.

Figura 1 - Taxa de detecção de sífilis em gestantes, Nordeste 2015-2019.

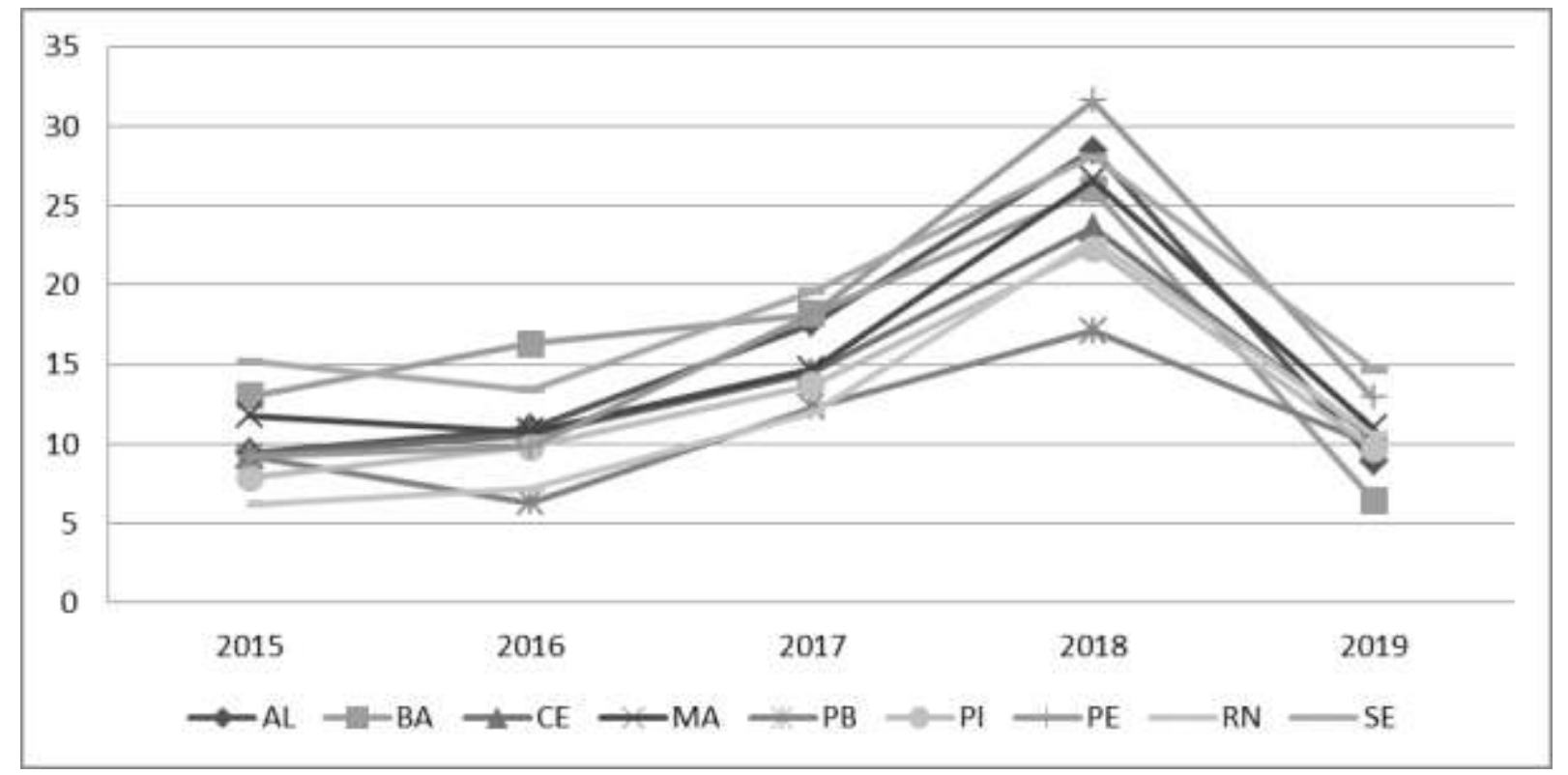

Fonte: SINAN/Ministério da Saúde/Brasil (2020).

\section{Discussão}

Durante os cinco anos de estudo, pôde-se perceber que a Sífilis atingiu uma população jovem e sexualmente ativa, compreendendo uma faixa etária de 20-29 anos (média de 51,3\%); em concordância com a literatura, este fato pode está relacionado a fatores determinantes e condicionantes de saúde como baixa escolaridade, acesso à educação em saúde, condições socioeconômicas, entre outros. A faixa entre 10 e 14 anos foi a que obteve menor número $(1,2 \%)$ em todos os anos estudados.

Um estudo realizado com 94 gestantes diagnosticadas com sífilis em Madrid constatou em seus resultados que as idades das pacientes estudadas se concentraram entre 15 e 45 anos, com uma média de 29 anos (Calle et al., 2013), já em outra pesquisa que abordou a temática natimortos e sífilis gestacional em países da América Latina, evidenciou-se que a maioria das gestantes estudadas tinham entre 20 e 34 anos de idade (Arnesen et al., 2015).

Notou-se nos resultados que as gestantes entre 10 e 14 anos foram aquelas com menores indicies, isto é positivo, porque sabe-se que a iniciação sexual precoce é considerada um comportamento de risco, pois o adolescente exposto a essa situação pode ter maiores chances de aumentar o número de parceiros sexuais durante a vida, até que o mesmo faça opção por relações monogâmicas estáveis e, quanto maior o número de parceiros sexuais, maior a chance de exposição as infecções sexualmente transmissíveis (ISTs) (Li et al., 2001, Tripp, Viner, 2005; Paiva et al., 2008; Batista, 2014).

Quando foram analisados os dados referentes à sífilis gestacional e escolaridade, observou-se que em 2018, 23,3\% das mulheres possuíam $5^{\mathrm{a}}$ a $8^{\mathrm{a}}$ série incompleta. Tal escolaridade é considerada baixa e sabe-se que a falta de acesso à educação favorece o adoecimento da população. 
A baixa escolaridade é comum a diferentes regiões do Brasil, isso compromete o acesso à educação em saúde, estratégia que possibilita a adesão a comportamentos saudáveis e mobilização social para melhorias nas condições de vida; além disso, influencia também na adesão a tratamentos, podendo representar dificuldades no entendimento das orientações realizadas (Andrade et al., 2014).

Em consonância ao presente estudo, uma pesquisa realizada em Palmas-TO que descreveu o perfil epidemiológico de sífilis gestacional e congênita, obteve também expressivo número em relação à baixa escolaridade entre as gestantes, sendo $76 \%$ possuidoras de ensino fundamental incompleto (Cavalcante; Pereira; Castro, 2017).

Outro estudo realizado em Caxias-MA também expressou em seus resultados altas taxas em relação à baixa escolaridade entre as gestantes com sífilis, sendo evidenciado que estas mulheres são moradoras de áreas precárias em assistência social para população (Conceição; Câmara; Pereira, 2019).

Quanto ao tipo de raça houve um predomínio do número de casos da raça parda, com 68,6\% no ano de 2018. As menores taxas ocorreram entre as mulheres indígenas com 0,4\% dos casos em 2017, o que pode está relacionado com o número de declarantes dessas raças no Brasil, pois de acordo com dados da Pesquisa Nacional por Amostra de Domicílios (PNAD) 2019, 42,7\% dos brasileiros se declararam como brancos, 46,8\% como pardos, 9,4\% como pretos e 1,1\% como amarelos ou indígenas (IBGE, 2019).

Com este estudo, pôde-se perceber que ao longo dos anos estudados, houve um aumento do número de casos de sífilis em todos os estados do nordeste brasileiro. Esse dado pode está relacionado ao fato da região nordeste historicamente ser a mais deficiente em termos socioeconômicos.

Em análise epidemiológica realizada no estado do Maranhão durante os anos de 2012 a 2017, também se constatou aumento nos casos de sífilis gestacional, o que pode apresentar uma urgência na realização de ações voltadas ao diagnóstico e tratamento da doença na população desta região, além da necessidade de orientações às mulheres de áreas mais precárias, onde o acesso à educação em saúde é ainda restrito (Magalhães et al., 2020).

Em estudo recente realizado em uma maternidade pública nordestina, os autores relataram que no Brasil, entre $2010 \mathrm{e}$ 2017, a taxa de detecção de sífilis em gestantes aumentou 4,9 vezes, passando de 3,5 para 17,2 casos por mil nascidos vivos, sendo a região Nordeste responsável por 16\% dos casos registrados em 2017 (Silva; Carvalho; Chaves, 2021).

Em relação à classificação, houve crescimento das taxas de sífilis secundária e latente, o que pode esta demonstrando falha no tratamento nos anos estudados. Quanto a variável realização do pré-natal, observou-se um predomínio das que realizaram em comparação com as que não realizaram.

Entretanto, ainda se faz necessário um acompanhamento ativo durante todo pré-natal. De acordo com pesquisa epidemiológica realizada em 2019, evidenciou-se que 94,17\% das crianças diagnosticadas com sífilis congênita nasceram de mães que realizaram o pré-natal (Favero et al., 2019).

Sabe-se também que o aumento gradativo de notificações de sífilis na assistência pré-natal tem forte influência da Rede Cegonha, que tem entre seus objetivos o aumento da cobertura de testes para as gestantes e o devido seguimento dos casos (Favero et al., 2019).

Comparando com o estudo de Silva et al (2021), os dados referentes ao diagnóstico e tratamento revelam que o diagnóstico foi realizado predominantemente durante o pré-natal, especificamente com maior percentual no $2^{\circ}$ trimestre de gestação (24,5\%); no entanto, houve um número importante de casos verificados durante o momento do parto na maternidade $(39,1 \%)$. Quanto à fase da doença em que se realizou o diagnóstico, 91,4\% das pacientes apresentavam sífilis latente (Silva; Carvalho; Chaves, 2021). Quando analisada a variável tratamento, pode-se perceber que o tratamento de escolha foi com a droga penicilina $\mathrm{G}$ benzatina e este tem se mostrado eficaz, de acordo com protocolo clínico institucionalizado pelo Ministério da Saúde (Brasil, 2015). 
Para Silva et al (2021), o tratamento com a penicilina G benzatina é o melhor método de prevenção da sífilis congênita, tanto da mãe como do seu parceiro sexual, o que caracteriza, em termos epidemiológicos, um bom indicador da qualidade da assistência pré-natal de uma população (Silva; Carvalho; Chaves, 2021).

Através de um estudo ecológico que analisou a incidência de sífilis e o tratamento na atenção básica durante o ano de 2014, foi possível evidenciar que em 41,9\% dos municípios brasileiros, mais de 50\% das equipes atuantes na atenção básica administram penicilina $\mathrm{G}$ benzatina em gestantes diagnosticadas com sífilis (Figueiredo et al., 2020).

Com relação ao momento do diagnóstico de sífilis, os resultados deste estudo apontam que houve um crescimento no número de casos quando o diagnóstico ocorreu durante o pré-natal (56,4\%), no ano de 2019. Esses dados são confortantes, pois sabe-se que o diagnóstico precoce, bem como o tratamento em tempo oportuno, podem reduzir ou eliminar as taxas de transmissão vertical. Essas taxas são ainda menores quando o momento do diagnóstico ocorreu no parto/curetagem com 33,5\% dos casos em 2018 e no pós-parto atingindo 5,4\% em 2019. Em uma pesquisa que descreveu os casos de sífilis gestacional e congênita em estados brasileiros no período de 2007 a 2012, também foi possível identificar altos indicies de diagnóstico durante o pré-natal, sendo equivalente a $74 \%$ da amostra. O diagnóstico durante o parto compreendeu-se em $18 \%$ dos casos (Saraceni et al., 2017).

Em relação à taxa de detecção da sífilis gestacional no período estudado, observou-se que 2018 foi o ano com maior número em todos os Estados do Nordeste, sendo o período entre 2015 a 2017 sem oscilações significativas, e o ano de 2019, o que registrou a menor taxa.

Um estudo epidemiológico que analisou as repercussões de uma modificação no código de saúde na cidade de Nova York, demonstrou em seus resultados um aumento significativo de mulheres grávidas infectadas com sífilis e/ou hepatite B em 2018, indicando que neste ano 91\% dos laboratórios avaliados certificaram alterações nos exames de gestantes (Liao et al., 2020).

Como limitações deste estudo têm-se a utilização de fontes secundárias para pesquisa, pois estas podem apresentar subnotificações e preenchimentos não adequados nas fichas de notificação acerca da sífilis gestacional no Sistema de Informação.

O estudo contribuiu para análise e discussão sobre a sífilis em gestantes na região Nordeste do Brasil. Os resultados foram capazes de expressar o perfil epidemiológico das gestantes, como também análise do tratamento utilizado e realização do pré-natal, reforçando a necessidade de uma assistência de qualidade para gestantes durante todo período gravídico.

\section{Conclusão}

Além de aprofundar os estudos acerca do perfil epidemiológico de sífilis gestacional na região nordeste do Brasil, este estudo pôde comprovar que durante os anos analisados (2015-2019) os casos de sífilis gestacional têm acometido em sua maioria mulheres jovens, sexualmente ativas, com baixa escolaridade, com predomínio da raça parda.

O tratamento de escolha entre os profissionais foi a penicilina, sendo este tratamento considerado adequado. Pôde-se notar também que, com o passar dos anos, houve um aumento gradativo do número de casos de sífilis nos estados do nordeste e que a doença tem sido diagnosticada precocemente durante as consultas de pré-natal, o que pode está contribuindo juntamente com o tratamento adequado, para uma redução dos casos de transmissão vertical e complicações fetais.

Sugere-se, portanto, que outros estudos científicos sejam realizados acerca da sífilis gestacional no Nordeste brasileiro, a fim de melhor identificar determinantes e/ou condicionantes que possam ser base para modificar a situação epidemiológica de saúde, seja dessa região, ou em outras do país. Seria válida uma comparação entre outras regiões do país para que houvesse uma maior riqueza de informações sobre o conteúdo abordado. 


\section{Referências}

Andrade, J. M. O., Rios, L. R, Teixeira, L. S., Vieira, F. S., Mendes, D. C., Vieira, M. A., \& Silveira, M. F. (2014). Influência de fatores socioeconômicos na qualidade de vida de idosos hipertensos. RevistaCiência\& Saúde Coletiva. 19(8), 3497-3504.

Arnesen, L., Martínez, G., Mainero, L., Serruya, S., \& Durán, P. (2015). Gestational Syphilis and Stillbirth in Latin America and the Caribbean.International Journal of Gynecology \& Obstetrics, 128(3), 241-45.

Batista, F. A. (2014). Comportamento sexual de risco em adolescentes escolares [dissertação]. Universidade Federal de Santa Maria, Centro de Ciências Sociais e Humanas.

Brasil, Ministério da Saúde, Sífilis. (2019). Boletim Epidemiológico. Brasília, Número Especial.

Brasil, Ministério da Saúde, Súfilis. (2020). Boletim Epidemiológico. Brasília, Número Especial.

Brasil, Ministério da Saúde. (2017). Secretaria de Vigilância em Saúde.Volume Único, 2ª Ed.Brasília: Ministério da Saúde.

Brasil, Ministério da Saúde. (2015). Secretaria de Vigilância em Saúde. Departamento de DST, Aids e Hepatites Virais. Protocolo Clínico e Diretrizes Terapêuticas para Atenção Integral às Pessoas com Infecções Sexualmente Transmissíveis.

Calle, M., Cruceyra, M., Haro, M., Magdaleno, F., Monteiro, M. D., Aracil, J., \& González, A. (2013). Sífilis y embarzo: estudio de 94 casos. Medicina Clínica, 14 (14), 141-144.

Cavalcante, P. A. M., Pereira, R. B. L., \& Castro, J. G. D. (2017). Sífilis gestacional e congênita em Palmas, Tocantins, 2007-2014. Epidemiologia e Serviços de Saúde, 26(2), 255-64.

Conceição, H., Câmara, J., \& Pereira, B. (2019). Análise Epidemiológica e Espacial dos casos de Sífilis gestacional e Congênita. Revista Saúde em Debate.

Favero, M. L. D. C., Ribas, K. A. W., Costa, M. C. D, \& Bonafe, S. M. (2019). Sifilis congênita e gestacional: notificação e assistência pré-natal. Arquivos de Ciências da Saúde. 26(1), 2.

Figueiredo, D. C. M. M., Figueiredo, A. M., Souza, T. K. B., Tavares, G, \& Vianna, R. P. T. (2020). Relação entre oferta de diagnóstico e tratamento da sífilis na atenção básica sobre a incidência de sífilis gestacional e congênita. Cadernos de Saúde Pública. 36(3).

IBGE. Instituto Brasileiro de Geografia e Eestatística. (2019). Informativo PNAD - Características gerais dos domicílios e dos moradores.

Liao, T. S., Hashmi, A, Lazaroff, J, Hennessy, R, Kim, A. S, Lioyd, P. E., \& Rosen, J. B. (2020). Effect of policy change to require laboratory reporting with pregnancy indicated for syphilis and hepatites B vírus infection, New York City. Public Health Reports, 135(1), 1825-188S.

Lima, T. M., Machado, I. L. L., Siqueira, J. P. Z., \& Almeida, M. T. G. (2019). Perfil Epidemiológico de Pacientes com Sífilis Congênita e Gestacional em um Município do Estado de São Paulo, Brasil. Revista Brasileira de Saúde Materno Infantil. 19(4): 873-880.

Li, X., Stanton, B., Cottrell, L., Burns, J., Pack, R., \& Kaljee, L. (2001). Patterns of initiation of sex and drug-related activities among urban low-income African-American adolescents.Journal of Adolescent Health. 28(1): 46-54.

Magalhães, L. M., et al (2020). Sífilis gestacional: impacto epidemiológico no estado do Maranhão, Brasil. Research, Society and Development, 9(2).

Oliveira, K. T. A., Morais, L. E., Leitão, J. M. S. R., Verde, R. M. C. L., Nascimento, M. H., Oliveira, T. R., Azevedo, P. S. S., Moraes, A. B, \& Oliveira, E. H. (2019). Caracterização da Sífilis em Gestantes no Município do Codó - Maranhão no período de 2012 a 2017 . Revista Eletrônica Acervo em Saúde.

Paiva, V., Calazans, G., Venturi, G., \& Dias, R. (2008). Idade e uso de preservativo na iniciação sexual de adolescentes brasileiros. Revista de Saude Pública. $1: 45-53$.

Saraceni, V, Pereira, G. F. M., Silveira, M. F., Araujo, M. A. L., \& Miranda, A. E. (2017). Vigilância Epidemiológica da transmissão vertical da sífilis: dados de seis unidades federativas no Brasil. Revista Panamericana de Salud Pública. 41.

Silva, N. C., Carvalho, K. B., \& Chaves, K. B. (2021). Sífilis gestacional em uma maternidade pública no interior do Nordeste brasileiro.Revista Femina. 49(1):58-64.

Souza, B. S. O., Rodrigues, R. M., \& Gomes, R. M. L. (2018). Análise Epidemiológica de Casos Notificados de Sífilis. Revista Sociedade Brasileira de Clínica Médica. 16(2): 94-8.

Terra, A. E. S. (2019). Caracterização Epidemiológica da Sífilis na Gestação em um Município do Interior do Brasil. Uberlândia, 35 p. Trabalho de Conclusão de Curso (Enfermagem). Universidade Federal de Uberlândia.

Tripp, J., \& Viner, R. (2005). Sexual health, contraception and teenage pregnancy. The BMJ. 330(7491):590-3. 